\title{
Complexidade Computacional de Problemas de Computar Medidas de Tendência Central e Dispersão com Entradas Intervalares
}

A.B. LORETO ${ }^{1}$, Departamento de Matemática, UNISC, 96815-900 Santa Cruz do Sul, RS, Brasil.

M.A. CAMPOS', Centro de Informática, UFPE, 50732-970 Recife, PE, Brasil.

D.M. CLAUDIO ${ }^{3}$, Faculdade de Matemática, PUCRS, 90619-900 Porto Alegre, RS, Brasil.

L.V. TOSCANI ${ }^{4}$, Instituto de Informática, UFRGS, 91501-970 Porto Alegre, RS, Brasil.

Resumo. O presente trabalho aborda a complexidade computacional dos problemas de computar, com entradas intervalares, as medidas de tendência central média, mediana e moda, e as medidas de dispersão amplitude total, variância, desvio padrão, coeficiente de variação, covariância e coeficiente de correlação. Para a investigação da complexidade elabora-se uma abordagem intervalar para os indicadores estatísticos e uma forma de representação dos valores reais em valores intervalares, de tal modo que não ocorram superestimação nos intervalos solução.

\section{Introdução}

Nas pesquisas realizadas sobre o tema complexidade de problemas de estatística descritiva com entradas intervalares, observou-se que não foram encontrados nenhum trabalho que tratasse sobre a complexidade computacional de problemas de medidas de tendência cental intervalar.

A literatura ([3], [4], [5]) mostra que foram realizadas pesquisas somente em problemas das medidas de dispersão variância, covariância e coeficiente de correlação com entradas intervalares, e que a utilização da computação intervalar na solução de problemas de medidas de dispersão sempre fornece solução com intervalos superestimados (intervalos com amplitude grande), e que ao procurar uma solução com

\footnotetext{
${ }^{1}$ abl@unisc.br;

${ }^{2}$ mac@cin.ufpe.br;

${ }^{3}$ dalcidio@inf.pucrs.br;

${ }^{4}$ laira@inf.ufrgs.br.
} 
intervalos de amplitude pequena (através da computação da imagem intervalar), o problema passa a pertencer a classe de problemas NP-Difícil.

Observa-se que a NP-dificuldade em problemas de medidas de dispersão com entradas intervalares está relacionada com o processamento dos dados de entrada (pois tem-se que considerar todos os valores compreendidos entre $\underline{x}$ e $\bar{x}$ do intervalo $\mathbf{x}$ para encontrar os extremos do intervalo solução). Outro fato importante, e que completa a caracterização dos problemas como NP-Difíceis, é que os problemas em questão são problemas de decisão, pois se deseja saber se existem intervalos $\mathbf{y}=[\underline{y}, \bar{y}]$ que contenham as soluções aproximadas destes problemas.

\section{Abordagem Intervalar de Medidas de Tendência Central e Dispersão}

Sejam $X$ e $Y$ variáveis aleatórias populacionais seguindo a lei da probabilidade $f_{X}\left(\theta_{X}\right)$ e $f_{Y}\left(\theta_{Y}\right)$, respectivamente. Sejam $\mu_{x}, \mu_{y}, \sigma_{x}^{2}$ e $\sigma_{y}^{2}$ as esperanças matemáticas e variâncias das variáveis aleatórias $X$ e $Y$. Adicionalmente, sejam $\left\{x_{1}, \ldots, x_{n}\right\}$ e $\left\{y_{1}, \ldots, y_{m}\right\}$ amostras aleatórias de $X$ e $Y$.

Uma forma de se considerar não somente os erros numéricos mas também os erros aleatórios inerentes aos valores $x_{1}, \ldots, x_{n}$ e $y_{1}, \ldots, y_{n}$ é considerar que, para $i=1, \ldots, n$ e $j=1, \ldots, n, x_{i} \in\left[\underline{x}_{i}, \bar{x}_{i}\right]$ e $y_{j} \in\left[\underline{y}_{j}, \bar{y}_{j}\right]$. Neste caso, consideram-se os dados de entrada $\left\{x_{1}, \ldots, x_{n}\right\}, x_{1} \in \mathbf{x}_{\mathbf{1}}, \ldots, x_{n} \in \mathbf{x}_{\mathbf{n}}$, ou seja, domínios intervalares onde $\mathbf{x}_{\mathbf{1}}=\left[\underline{x}_{1}, \bar{x}_{1}\right], \ldots, \mathbf{x}_{\mathbf{n}}=\left[\underline{x}_{n}, \bar{x}_{n}\right]$. Nestas situações, para diferentes valores possíveis $x_{i} \in \mathbf{x}_{\mathbf{i}}$, obtém-se diferentes valores da média me, mediana $m d$, moda $m o$, amplitude total at, variância $v a$, desvio padrão $d p$, coeficiente de variação $c v$, covariância $c o$ e coeficiente de correlação $c c$.

Considerando que tem-se dados de entrada intervalares, deseja-se conhecer quais são os intervalos da média intervalar $\mathbf{M} \mathbf{E}_{v}$, mediana intervalar $\mathbf{M} \mathbf{D}_{v}$, moda intervalar $\mathbf{M O}_{v}$, amplitude total intervalar $\mathbf{A} \mathbf{T}_{\mathbf{v}}$, variância intervalar $\mathbf{V A}{ }_{v}$, desvio padrão intervalar $\mathbf{D P}{ }_{v}$, coeficiente de variação intervalar $\mathbf{C V} v$, covariância intervalar $\mathbf{C O} v$ e coeficiente de correlação intervalar $\mathbf{C} \mathbf{C}_{v}$.

A seguir apresenta-se uma abordagem intervalar para os indicadores estatísticos citados anteriormente:

- Média aritmética Intervalar: $\mathbf{M E}_{v}=[\underline{m e}, \overline{m e}]=\frac{1}{n-1}\left[\sum_{i=1}^{n} \underline{x}_{i}, \sum_{i=1}^{n} \bar{x}_{i}\right]$.

- Mediana Intervalar: $\mathbf{M D}_{v}= \begin{cases}\left(\mathbf{x}_{\left(\frac{n}{2}\right)}+\mathbf{x}_{\left(\frac{n}{2}+1\right)}\right) / 2, & \text { se } n \text { for par } \\ \left(\mathbf{x}_{\left(\frac{n+1}{2}\right)}\right), & \text { se } n \text { for ímpar. }\end{cases}$

- Moda Intervalar: Se existe um valor modal para os dados reais, então

$$
\mathbf{M O}_{v}=[\underline{m o}, \overline{m o}]=\left[m o\left\{\underline{x}_{i}\right\}, m o\left\{\bar{x}_{i}\right\}\right], 1 \leq i \leq n .
$$

- Amplitude total Intervalar: $\mathbf{A T}_{v}= \begin{cases}{[\underline{m a}-\overline{m i}, \overline{m a}-\underline{m i}],} & \text { se } \underline{m a} \geq \overline{m i} \\ {[0, \overline{m a}-\underline{m i}],} & \text { se } \underline{m a}<\overline{m i} .\end{cases}$ onde $M I=[\underline{m i}, \overline{m i}]=\left[\min _{1 \leq i \leq n}\left\{\underline{x}_{i}\right\}, \min _{1 \leq i \leq n}\left\{\bar{x}_{i}\right\}\right]=$, é o Mínimo Interva- 
lar e $M A=[\underline{m a}, \overline{m a}]=\left[\max _{1 \leq i \leq n}\left\{\underline{x}_{i}\right\}, \max _{1 \leq i \leq n}\left\{\bar{x}_{i}\right\}\right]$ é o Máximo Intervalar.

- Variância Intervalar: $\mathbf{V A}_{v}=[\underline{v a}, \overline{v a}]=\frac{1}{n-1} \sum_{i=1}^{n}\left(\mathbf{x}_{\mathbf{i}}-\mathbf{M E}\right)^{2}$.

- Desvio padrão Intervalar: $\mathbf{D P}_{v}=[\underline{d p}, \overline{d p}]=+\sqrt{[\underline{v a}, \overline{v a}]}=[+\sqrt{\underline{v a}},+\sqrt{\overline{v a}}]$.

- Coeficiente de variação Intervalar: Se $0 \notin[\underline{m e}, \overline{m e}]$, então

$\mathbf{C V}_{v}=[\underline{c v}, \overline{c v}]=\frac{\mathbf{D P}}{\mathbf{M E}}=\frac{[d p, \overline{d p}]}{[\underline{m e}, \overline{m e}]}$

- Covariância Intervalar: $\mathbf{C O}_{v}=[\underline{c o}, \overline{c o}]=\frac{1}{n-1} \sum_{i=1}^{n}\left(\mathbf{x}_{\mathbf{i}}-\mathbf{M E}_{\mathbf{X}}\right)\left(\mathbf{y}_{\mathbf{i}}-\mathbf{M E}_{\mathbf{Y}}\right)$,

onde $\mathbf{M E}_{\mathbf{X}}$ é a média intervalar dos valores de $X$ e $\mathbf{M} \mathbf{E}_{\mathbf{Y}}$ a média intervalar dos valores de $Y$.

- Coeficiente de correlação Intervalar: Se $0 \notin \mathbf{D P}_{\mathbf{X}} \mathbf{D P}_{\mathbf{Y}}$, então

$\mathbf{C C}_{v}=[\underline{c c}, \overline{c c}]=\frac{\mathbf{C O}}{\mathbf{D P}_{\mathbf{X}} \mathbf{D P}_{\mathbf{Y}}}$, onde $\mathbf{C O}$ é a covariância intervalar, $\mathbf{D P}_{\mathbf{X}}$ o desvio padrão intervalar dos valores de $X$ e $\mathbf{D P}_{\mathbf{Y}}$ o desvio padrão intervalar dos valores de $Y$.

Observação: Para calcular a mediana os dados devem estar ordenados em ordem ascendente. Levando em consideração que os dados intervalares $\mathbf{x}_{\mathbf{1}}, \ldots, \mathbf{x}_{\mathbf{n}}$ podem ser todos disjuntos ou todos encaixados, ordena-se os dados intervalares de acordo com os seguintes casos:

- Todos os intervalos disjuntos: considera-se a ordem intervalar de Kulisch [9]: $\mathbf{x}_{\mathbf{1}} \leq \mathbf{x}_{\mathbf{2}} \leq \ldots \leq \mathbf{x}_{\mathbf{n}}$, onde $\mathbf{x}_{\mathbf{1}} \leq \mathbf{x}_{\mathbf{2}} \Longleftrightarrow \underline{x}_{1} \leq \underline{x}_{2} \wedge \bar{x}_{1} \leq \bar{x}_{2} ;$

- Todos os intervalos encaixados: neste caso, para a ordenação dos elementos, considera-se a ordem de inclusão $\mathbf{x}_{(\mathbf{1})} \subseteq \mathbf{x}_{(\mathbf{2})} \subseteq \ldots \subseteq \mathbf{x}_{(\mathbf{n})}$ onde

$\mathbf{x}_{(\mathbf{1})} \subseteq \mathbf{x}_{(\mathbf{2})} \Longleftrightarrow \underline{x}_{1} \geq \underline{x}_{2} \wedge \bar{x}_{1} \leq \bar{x}_{2}[11]$

As definições intervalares elaboradas para os indicadores estatísticos, média, moda, variância, desvio padrão, coeficiente de variação, covariância e coeficiente de correlação, suportam dados intervalares todos disjuntos ou todos encaixados.

A partir das expressões intervalares definidas para os indicadores estatísticos, propõe-se algoritmos para a solução dos problemas de computar os intervalos de medidas de tendência central intervalar e dispersão intervalar. Tais algoritmos utilizam a aritmética intervalar definida por Moore [11] e a extensão intervalar $[12]$. 


\section{Técnicas de Computação Intervalar}

Na computação intervalar, pode-se calcular o intervalo solução $\mathbf{y}=[y, \bar{y}]$ através de métodos de aproximação, técnicas de otimização e extensão intervalār.

Dentre os métodos de aproximação existentes, cita-se o Método de Linearização de uma função. Quando é suficiente obter os valores aproximados dos extremos $\underline{y}$ e $\bar{y}$ do intervalo $\mathbf{y}$, pode-se linearizar a função $f\left(x_{1}, \ldots, x_{n}\right)$, isto é, representar $x_{i}$ como $x_{i}=\widetilde{x}_{i}-\Delta x_{i}$, e expandir a expressão resultante $f\left(x_{1}, \ldots, x_{n}\right)=$ $f\left(\widetilde{x}_{1}-\Delta x_{1}, \ldots, \widetilde{x}_{n}-\Delta x_{n}\right)$ em série de Taylor em relação a $\Delta x_{i}$, omitindo termos quadráticos e de ordem superior nesta expansão. Para a função linear resultante (aproximada), calcula-se o intervalo y da seguinte maneira: i) $\underline{y}=\widetilde{y}-\left|a_{1}\right| \cdot \Delta_{1}-$ $\ldots-\left|a_{n}\right| \cdot \Delta_{n}$ e ii) $\bar{y}=\tilde{y}+\left|a_{1}\right| \cdot \Delta_{1}+\ldots+\left|a_{n}\right| \cdot \Delta_{n}$.

Segundo Kreinovich et al. [8], para muitos problemas práticos métodos de aproximação não são suficientes, pois deve-se ter uma estimativa garantida para o intervalo y. Esta estimativa não é garantida devido a uma série de fatores como: i) medições de erros relativamente grandes, de modo que seus quadrados não podem ser omitidos seguramente; ii) a função $f\left(x_{1}, \ldots, x_{n}\right)$ que descreve a relação entre quantidades medidas diretamente $x_{i}$ e a quantidade desejada $y$, pode não ser linear; iii) existem problemas nos quais necessita-se de uma estimativa garantida, pois uma superestimação poder ser desastrosa.

Uma das técnicas de otimização é o cálculo do ponto de máximo e de mínimo de uma função, também conhecido como Imagem Intervalar [12]. O problema de encontrar os extremos do intervalo solução é um problema de otimização: o extremo inferior $y$ é a solução do problema de minimização $f\left(x_{1}, \ldots, x_{n}\right) \rightarrow$ min sobre as condições $\underline{x}_{i} \leq x_{i} \leq \bar{x}_{i}, 1 \leq i \leq n$ (onde $\underline{x}_{i}=\widetilde{x}_{i}-\Delta_{i}$ e $\bar{x}_{i}=\widetilde{x}_{i}+\Delta_{i}$ ), $\mathrm{e}$ o extremo superior $\bar{y}$ é a solução do problema de maximização $f\left(x_{1}, \ldots, x_{n}\right) \rightarrow$ max sobre as condições $\underline{x}_{i} \leq x_{i} \leq \bar{x}_{i}, 1 \leq i \leq n$. Calcular o valor $f(x)$ de todos os pontos "candidatos" e de todos os extremos significa encontrar todos os valores de $f(x)$ de todos estes pontos, o maior valor de $f(x)$ é o máximo desejado (correspondentemente, o menor dos valores de $f(x)$ é o mínimo desejado). Para uma aplicação proveitosa desta técnica deve-se considerar o tipo de função, se a função $f(x)$ é muito complicada, então a equação $d f / d x=0$ também será muito complicada e, por essa razão, difícil de resolver, porém para funções razoavelmente simples este método é muito eficiente.

Ratschek [13] afirma que a maioria dos métodos de otimização carregam no mínimo dois defeitos (falhas): i) o método não garante que os pontos de mínimo possam ser encontrados para uma dada tolerância e ii) dependendo das condições da função, permite encontrar somente o mínimo local ao invés do global. Estes defeitos dificultam a solução de problemas de otimização global. A otimização global é considerada, por essa razão, um assunto intratável.

Segundo Ferson et al. [4], históricamente o primeiro método para computar o intervalo solução é o método o qual chamamos de extensão intervalar [12]. Na extensão intervalar, repetimos a computação formando o programa $f$ passo-a-passo, substituindo cada operação elementar de números reais pela correspondente operação da aritmética intervalar [12]. Em alguns casos o intervalo solução é exato, porém outros casos mais complexos (como o problema de computar o intervalo da 
variânica, por exemplo) obtém-se intervalos solução superestimados.

Dentre os métodos citados acima, escolhe-se a extensão intervalar por se adequar às expressões dos indicadores estatísticos com abordagem intervalar (definidos anteriomente), e por tornar mais acessível a projeção de algoritmos imediatos (algoritmos de fácil construção) [14].

\section{Complexidade Computacional de Problemas}

O termo complexidade, no contexto de algoritmos, refere-se aos requerimentos de recursos necessários para que um algoritmo possa resolver um problema sob o ponto de vista computacional, ou seja, à quantidade de trabalho despendido pelo algoritmo [14]. Quando o recurso é o tempo, são escolhidas uma ou mais operações fundamentais e então são contados os números de execuções desta operação fundamental na execução do algoritmo. Segundo Toscani [14] a escolha de uma operação como operação fundamental é aceitável se o número de operações executadas pelo algoritmo é proporcional ao número de execuções da operação fundamental.

A complexidade também pode ser vista como uma propriedade do problema, o que significa dar uma medida independente do tratamento dado ao problema, independente do caminho percorrido na busca da solução, portanto independente de algoritmos. Alguns problemas são bem comportados, isto é, permitem chegar a limites de complexidades bem definidos, outros estão em classes com contornos não bem claros [14].

Quanto a complexidade do problema, são definidas várias classes, como: P, NP, NP-Completo e NP-Difícil, entre outras. Um problema (da classe P) é considerado tratável se existe um algoritmo determinístico de tempo polinomial que resolve todas as instâncias deste. Um problema é dito intratável se a sua complexidade inferior (melhor algoritmo possível) não é polinomial [14]. Para um problema pertencer a classe NP significa que existe pelo menos um algoritmo não-determinístico que o resolva em tempo polinomial. Um problema (não necessariamente da classe NP) é chamado NP-Difícil se todo o problema da classe NP pode ser reduzido a este. Se um problema da classe NP é NP-Difícil, este pode ser chamado de NP-Completo. Para problemas das classes NP-Completo e NP-Difícil não se espera encontrar um algoritmo eficiente para verificá-lo. Qualquer problema NP-Difícil pertencente ou não à classe $\mathrm{NP}$, tem a propriedade de não ser resolvido em tempo polinomial, a menos que $\mathrm{P}=\mathrm{NP}[6][8]$.

Identificar a tratabilidade e a intratabilidade dos problemas, mesmo aqueles que possuem algoritmos imediatos [14], é de extrema importância para os projetistas de algoritmos, pois conhecendo as classes de complexidade a que os problemas pertencem, os projetistas poderiam ter uma medida real quanto às soluções disponíveis e a expectativa de melhorar esses resultados. 


\subsection{Análise da Complexidade dos Problemas de Medidas de Tendência Central e Dispersão}

Para a investigação da complexidade de problemas de medidas de tendência central intervalar e dispersão intervalar definimos um novo problema, para cada indicador estatístico descritivo intervalar, através da definição do domínio intervalar de funções intervalares com variáveis intervalares.

$\mathrm{Na}$ literatura, os problemas dos indicadores estatísticos variância, covariância e coeficiente de variação são definidos considerando variáveis reais $x$ e $y, n$ valores reais $x_{1}, \ldots, x_{n}$ (onde $x_{1} \in \mathbf{x}_{\mathbf{1}}, \ldots, x_{n} \in \mathbf{x}_{\mathbf{n}}$ ), $n$ valores reais $y_{1}, \ldots, y_{n}$ (onde $y_{1} \in$ $\left.\mathbf{y}_{\mathbf{1}}, \ldots, y_{n} \in \mathbf{y}_{\mathbf{n}}\right)$ e, como domínio, intervalos $\mathbf{x}_{\mathbf{i}}$ contidos no domínio da função $f\left(x_{1}, \ldots, x_{n}\right), x_{1} \in \mathbf{x}_{\mathbf{1}}, \ldots, x_{n} \in \mathbf{x}_{\mathbf{n}}$, ou seja, $\mathbf{x}_{\mathbf{i}} \subset \operatorname{Dom}(f)$. A busca da solução destes problemas é através da computação da imagem intervalar.

No presente trabalho considera-se, para os problemas de medidas de tendência central e dispersão, variáveis intervalares $\mathbf{x}$ e $\mathbf{y}, n$ valores intervalares $\mathbf{x}_{\mathbf{1}}, \ldots, \mathbf{x}_{\mathbf{n}}$, $n$ valores intervalares $\mathbf{y}_{\mathbf{1}}, \ldots, \mathbf{y}_{\mathbf{n}}$ e domínio composto por valores intervalares, ou seja, $f\left(\mathbf{x}_{1}, \ldots, \mathbf{x}_{\mathbf{n}}\right), \mathbf{x}_{\mathbf{i}}=\operatorname{Dom}(f) \subseteq \Re$. Para a solução destes problemas, aplica-se a extensão intervalar [12].

Por meio da análise da complexidade computacional verificamos que os problemas de medidas de tendência central intervalar e dispersão intervalar pertencem à classe de problemas P.

A Tabela 1 apresenta a comparação entre os resultados de complexidade dos problemas dos indicadores estatísticos descritivos considerando a utilização da extensão intervalar (nossos resultados) e a imagem intervalar (estado da arte). Utilizamos o símbolo "-" para indicar que não foram encontrados resultados de complexidade para os referidos problemas intervalares.

\begin{tabular}{lcc}
\hline $\begin{array}{c}\text { Problemas } \\
\text { dos Indicadores } \\
\text { Intervalares }\end{array}$ & $\begin{array}{c}\text { Complexidade } \\
\text { dos Problemas } \\
\text { com Extensão } \\
\text { Intervalar }\end{array}$ & $\begin{array}{c}\text { Complexidade } \\
\text { dos Problemas } \\
\text { com Imagem } \\
\text { Intervalar }\end{array}$ \\
\hline Média & $\mathrm{P}$ & - \\
Mediana & $\mathrm{P}$ & - \\
Moda & $\mathrm{P}$ & - \\
Amplitude Total & $\mathrm{P}$ & - \\
Variância & $\mathrm{P}$ & NP-Difícil \\
Desvio Padrão & $\mathrm{P}$ & - \\
Coef. de Variação & $\mathrm{P}$ & - \\
Covariância & $\mathrm{P}$ & NP-Difícil \\
Coef. de Correlação & $\mathrm{P}$ & NP-Difícil \\
\hline
\end{tabular}

Tabela 1: Problemas dos indicadores intervalares, complexidade dos problemas com extensão intervalar e com imagem intervalar.

\section{Verificação da Superestimação}

Segundo Ratschek et al. [13], os computadores utilizam a aritmética de ponto flutuante (padrão IEEE). Nesta aritmética números reais são aproximados por um 
subconjunto de números reais chamados representação numérica da máquina. Devido esta representação são gerados dois tipos de erros: i) ocorre quando uma entrada de valor real é aproximada por um número de máquina e ii) é causado por resultados intermediários aproximados pelos números de máquina. A aritmética intervalar fornece uma ferramenta para estimar e controlar esses erros automaticamente. No lugar de aproximar um valor real $x$ por um número de máquina, o valor real $x$, usualmente desconhecido, é aproximado por um intervalo $\mathbf{x}$ tendo número de máquina nos extremos inferior e superior. O intervalo $\mathrm{x}$ contém o valor $x$. $\mathrm{O}$ comprimento (ou diâmetro) deste intervalo pode ser usado como medida para qualidade da aproximação. Os cálculos são executados usando intervalos no lugar de números reais e, conseqüentemente, a aritmética real é substituída pela aritmética intervalar. Os erros, na computação intervalar, podem ser estimados por erro absoluto: $|x-m(\mathbf{x})|<w(\mathbf{x}) / 2$, onde $w(\mathbf{x})=\bar{x}-\underline{x}$ é o diâmetro do intervalo $\mathbf{x}$, e por erro relativo: $\left|\frac{x-m(\mathbf{x})}{x}\right| \leq \frac{w(\mathbf{x})}{2 m i n|\mathbf{x}|}$ se $0 \notin \mathbf{x}$.

Para certificação da não ocorrência de superestimação nos intervalos solução, apresentam-se exemplos de cálculos intervalares usando sistema de ponto flutuante e arredondamento direcionado [9].

Os valores reais $\left\{x_{1}, \ldots, x_{n}\right\}$, das amostras aleatórias de uma população, são representados por intervalos $\mathbf{x}_{\mathbf{i}}=\left[x_{i}-\delta, x_{i}+\delta\right]$, onde $\delta$ é a precisão escolhida para os valores reais.

Na verificação da superestimação no intervalo solução, deve-se considerar o valor $x_{k} \in \Re$, o intervalo $\mathbf{x}_{\mathbf{k}}=\left[\underline{x}_{k}, \bar{x}_{k}\right]$ e uma dada exatidão $\varepsilon$. A análise do erro é realizada através das seguintes medidas de erros absolutos:

- i) $\left|x_{k}-m\left(\mathbf{x}_{\mathbf{k}}\right)\right|<\varepsilon$, onde $m\left(\mathbf{x}_{\mathbf{k}}\right)=\left(\underline{x}_{k}+\bar{x}_{k}\right) / 2$ é o ponto médio do intervalo $\mathbf{x}_{\mathbf{k}}$;

- ii) $\left|x_{k}-m\left(\mathbf{x}_{\mathbf{k}}\right)\right|<w\left(\mathbf{x}_{\mathbf{k}}\right) / 2$, onde $w\left(\mathbf{x}_{\mathbf{k}}\right)=\bar{x}_{k}-\underline{x}_{k}$ é o diâmetro do intervalo $\mathbf{x}_{\mathbf{k}}$.

Exemplificando, consideramos a altura de uma turma de 21 alunos da 5a. série da escola Imaculada Conceição, localizada na cidade de Cachoeira do Sul, RS: $\{1.44$, $1.31,1.53,1.45,1.51,1.43,1.46,1.42,1.40,1.41,1.49,1.47,1.50,1.60,1.49,1.53$, $1.51,1.60,1.48,1.32,1.46\}$. Representamos estes valores em intervalos com precisão $\delta=0.005:\{[1.435,1.445],[1.305,1.315],[1.525,1.535],[1.445,1.455],[1.505,1.515]$, $[1.425,1.435],[1.455,1.465],[1.415,1.425],[1.395,1.405],[1.405,1.415],[1.485$, $1.495],[1.465,1.475],[1.495,1.505],[1.595,1.605],[1.485,1.495],[1.525,1.535]$, $[1.505,1.515],[1.595,1.605],[1.475,1.485],[1.315,1.325],[1.455,1.465]\}$.

Para calcular os indicadores estatísticos covariância e coeficiente de correlação precisa-se de dois conjuntos de dados. Dessa forma, considera-se a altura de 21 alunos da turma da $6 \mathrm{a}$. série da mesma escola citada anteriormente: $\{1.48,1.44$, $1.50,1.60,1.41,1.65,1.55,1.56,1.54,1.55,1.53,1.51,1.68,1.49,1.38,1.50,1.48$, $1.42,1.56,1.56,1.55\}$. Representamos estes valores em intervalos com a mesma precisão $\delta=0.005:\{[1.475,1.485],[1.435,1.445],[1.495,1.505],[1.595,1.605]$, $[1.405,1.415],[1.645,1.655],[1.545,1.555],[1.555,1.565],[1.535,1.545],[1.545$, $1.555],[1.525,1.535],[1.505,1.515],[1.675,1.685],[1.485,1.495],[1.380,1.390]$, 
$[1.495,1.505],[1.475,1.485],[1.415,1.425],[1.555,1.565],[1.555,1.565],[1.545$, $1.555]\}$.

Os cálculos dos indicadores estatísticos intervalares foram realizados utilizando sistema de ponto flutuante $\mathrm{F}(10,4,-10,10)$ com arredondamento direcionado [9]. Como exatidão, na medida do erro, considera-se $\varepsilon=10^{-2}$.

$\mathrm{Na}$ Tabela 2 apresentam-se os resultados dos indicadores estatísticos com dados reais e dados intervalares.

\begin{tabular}{lcc}
\hline \multicolumn{1}{c}{ Problemas } & Dados & Dados \\
dos Indicadores & Reais & {$[1.5352,1.5458]$} \\
Média & 1.5405 & {$[1.465,1.475]$} \\
Mediana & 1.47 & {$[1.455,1.465],[1.485,1.495]$,} \\
Moda & $1.46,1.49$, & {$[1.595,1.605]$} \\
& $1.51,1.53$, & {$[0.28,0.30]$} \\
Amplitude Total & 1.60 & {$[0.009339,0.01299]$} \\
Variância & 0.29 & {$[0.09663,0.1139]$} \\
Desvio Padrão & 0.01105 & {$[0.06251,0.07422]$} \\
Coef. de Variação & 0.1051 & {$[0.002688,0.006439]$} \\
Covariância & 0.06824 & {$[0.2027,0.6769]$} \\
Coef. de Correlação & 0.004495 & \\
\hline
\end{tabular}

Tabela 2: Problemas dos indicadores, dados reais e dados intervalares.

Na Tabela 3 apresentamos os erros obtidos ao calcularmos os indicadores estatísticos com valores intervalares.

\begin{tabular}{lcc}
\hline \multicolumn{1}{c}{ Problemas } & Erros & Absolutos \\
dos Indicadores & $(\mathrm{i})$ & $0<0.005250$ \\
\hline Média & 0.005250 & $0<0.005$ \\
Mediana & 0 & $0<0.005$ \\
Moda & 0 & $0<0.01$ \\
Amplitude Total & 0 & $0.0001103<0.001823$ \\
Variância & 0.001933 & $0.0001681<0.008656$ \\
Desvio Padrão & 0.008824 & $0.0001291<0.005852$ \\
Coef. de Variação & 0.005981 & $0.00006823<0.001876$ \\
Covariância & 0.001944 & $0.04229<0.2371$ \\
Coef. de Correlação & 0.2793 & \\
\hline
\end{tabular}

Tabela 3: Problemas dos indicadores e erros absolutos.

Os cálculos reais dos indicadores estatísticos foram realizados no software NetBook [2]. Os cálculos intervalares foram realizados no software Maple Intervalar [7].

\section{Considerações Finais}

As pesquisas desenvolvidas estão concentradas em alguns indicadores estatísticos como variância e covariância, talvez por serem os indicadores mais utilizados ou mais comuns na área de estatística. 
Conforme comentado anteriormente, e descrito na literatura, a utilização da computação intervalar para calcular o intervalo da variância, covariância e coeficiente de correlação sempre fornece intervalos superestimados (intervalos com amplitude grande), e que ao utilizar a imagem intervalar os problemas destes indicadores estatísticos passam a pertencer a classe de problemas NP-Difícil.

Por não existir na literatura uma abordagem intervalar da estatística descritiva (existe apenas para alguns indicadores descritivos), o presente trabalho aborda a nível intervalar, praticamente, todos os indicadores estatísticos descritivos.

Preocupados com os resultados de NP-Dificuldade obtidos para os problemas de computar os intervalos da variância, covariância e coeficiente de correlação, concentramos nossas pesquisas em métodos da computação intervalar que tornasse possível a computação dos intervalos destes indicadores, e dos demais como média, mediana, moda, amplitude total, desvio padrão e coeficiente de variação.

No trabalho desenvolvido, observa-se que: para os indicadores média intervalar e amplitude total intervalar, o valor intervalar é o mesmo se considerar a computação da imagem intervalar ou a extensão intervalar, ou seja, não existe intervalo com problema de superestimação; para os indicadores moda intervalar e mediana intervalar não necessitamos utilizar métodos de computação intervalar, pois não apresentam operações aritméticas entre intervalos. Os valores intervalares para este indicador são os intervalos dos dados de entrada, não ocorrendo problema de intervalos superestimados; os indicadores variância, covariância e coeficiente de correlação (analisados na literatura) tornam-se possíveis de serem calculados utilizando a extensão intervalar. Para estes indicadores verificamos a não ocorrência de superestimação nos intervalos calculados, fato este verificado nos cálculos realizados e apresentados nas Tabelas 2 e 3; o desvio padrão e o coeficiente de variação, que antes não eram possíveis de serem calculados devido a variância ser um problema NP-Difícil, são calculados através de operações aritméticas intervalares. Os valores intervalares obtidos não são superestimados, conforme Tabelas 2 e 3 . A qualidade de um intervalo está relacionada com o diâmetro do mesmo [13]. A verificação da ocorrência de intervalos superestimados dá-se justamente para analisar a qualidade do intervalo solução. Para garantir a qualidade de um intervalo, ou a não ocorrência de intervalos superestimados, representamos os valores reais de uma amostra em valores intervalares considerando uma margem de precisão $\delta$.

Abstract. The present work presents the computational complexity of problems to compute, with input intervals, the measures of central trend, average, medium and mode, and the measures of dispersion total amplitude, variance, standard deviation, coefficient variation, covariance and coefficient of correlation. For the inquiry of the complexity one is elaborated interval boarding for the statistical pointers and a form of representation of the real values in values intervals, of such way that does not occur overestimation in the solution intervals.

\section{Referências}

[1] M.A. Campos, R.A. Faria, Definição de alguns indicadores estatísticos usando intervalos, em "XI Congresso Nacional de Matemática Aplicada e Computacional", SBMAC, Ouro Preto, MG, 1988. 
[2] M.A. Campos, E.L. Silva, D.C. Pedrosa, J.A. Loureiro, J.L.C. Silva, C.A. Ferraz, NetBook: uma ferramenta para avaliação de desempenho em redes de comunicação, em "Salão de Ferramentas, Simpósio Brasileiro de Redes de Computadores", pp. 967-974, 2004.

[3] S. Ferson, L. Ginzburg, V. Kreinovich, L. Longpré, M. Aviles, Exact bounds on sample variance of interval data, em "Proc. Extended Abstracts of the 2002 SIAM Workshop on Validated Computing", pp. 67-69, Toronto, Canada, 2002.

[4] S. Ferson, L. Ginzburg, V. Kreinovich, J. Lopez, Absolute Bbounds on the mean of sum, product, etc.: a probabilistic extension of interval arithmetic, em "Proc. Extended Abstracts of the 2002 SIAM Workshop on Validated Computing", pp. 70-72, Toronto, Canada, 2002.

[5] S. Ferson, L. Ginzburg, V. Kreinovich, L. Longpré, M. Aviles, Computing Variance for Interval Data is NP-Hard, ACM SIGACT News, 33 No. 2 (2002), $108-118$.

[6] M.E. Garey, D.S. Johnson, "Computers and Intractability: a guide to the theory of NP-completeness", Freeman, San Francisco, 1979.

[7] H. Grimmer, Interval arithmetic in Maple with intpakX, To appear in: "PAMM - Proceedings in Applied Mathematics and Mechanics", GAMM-Conference Augsburg 2002, Wiley-InterScience.

[8] V.Kreinovich, "Computational Complexity and Feasibility of Data Processing and Interval Computations", KLUWER, 1998.

[9] U.W. Kulisch, W.L. Miranker, "Computer Arithmetic in Theory and Practice", Academic Press, New York, 1981.

[10] S. Lipschutz, "Probabilidade", McGraw-Hill, São Paulo, 1972.

[11] R.E. Moore, "Interval Analysis", Prentice-Hall, Englewood Cliffs, New Jersey, 1966.

[12] R.E. Moore, "Methods and Applications of Interval Analysis", Society for Industrial and Applied Mathematics, Philadelphia, PA, USA, 1979.

[13] H. Ratschek, J. Rokne, "New Computer Methods for Global Optimization", Ellis Horwood Limited, Great Britain, 1988.

[14] L.V. Toscani, P.A. Veloso, "Complexidade de Algoritmos: análise, projetos e métodos", Sagra-Luzzato, Porto Alegre, Instituto de Informática da UFRGS, 2001. 